\title{
Color Laplacian and Color Signless Laplacian Energy of Complement of Subgroup Graph of Dihedral Group
}

\author{
$1^{\text {st }}$ Abdussakir ${ }^{1}, 2^{\text {nd }}$ Mohammad Nafie Jauhari ${ }^{1}, 3^{\text {rd }}$ Nabila Umar ${ }^{1}, 4^{\text {th }}$ Lila Aryani \\ Puspitasari $^{1}$ \\ \{sakir,nafie.jauhari@mat.uin-malang.ac.id¹,15610120@student.uin-malang.ac.id¹, \\ 15610107@student.uin-malang.ac.id ${ }^{1}$ \}
}

UIN Maulana Malik Ibrahim, Department of Mathematics, Malang, Indonesia ${ }^{1}$

\begin{abstract}
Laplacian and signless laplacian energy of a finite graph is the most interesting topics on areas of energy of a graph. The new concept of energy of a graph is color energy and furthermore color laplacian and color signless laplacian energy. In this paper, the formulae of color laplacian and color signless laplacian energy of complement of subgroup graphs of dihedral group are determined. Color laplacian and color signless laplacian spectrum of these graphs are also computed.
\end{abstract}

Keywords: Color Laplacian Energy, Color Signless Laplacian enerdy, Subgroup Graph, Dihedral Group

\section{Introduction}

The energy of a finite graph $G$ is defined as the sum of absolute values of all eigenvalues of matrix of $G$ (Balakrishnan, 2004). Gutman (1978) introduced the concept of adjacency energy of a graph, and now it has evolved into other concept of energy. Researches related to the energy of a graph have been done, such as adjacency energy (Gutman, 2001), incidence energy (Gutman et al., 2009 and Jooyandeh et al., 2009), Harary energy (Güngör \& Çevik, 2010), Randic energy (Das et al., 2014), maximum degree energy (Adiga \& Smitha, 2009), detour energy (Ayyaswamy \& Balachandran, 2010), matching energy (Gutman \& Wagner, 2012), distance energy (Ramane et al., 2008), covering distance energy (Kanna et al., 2013), dominating distance energy (Kanna et al.. 2014), Laplacian energy (Gutman \& Zhou, 2006, Zhou \& Gutman, 2007 and Zhou et al.. 2008) and signless Laplacian energy (Liu, 2010). Subsequent developments introduced the concept of color energy of graph (Adiga et al.. 2013) and finally color Laplacian (Bhat \& D'souza, 2015) and color signless Laplacian energy (Bhat \& D'Souza, 2017a) of graph.

Graphs obtained from a group have also been introduced, such as Cayley graph (Heydemann, 1997), transitive Cayley graph (Kelarev \& Praeger, 2003), conjugate graph (Erfanian \& Tolue, 2012), commuting graphs (Chelvam et al., 2011), non-commuting graphs (Raza \& Faizi, 2013), inverse graphs (Alfuraidan \& Zakariya, 2017), identity graphs (Kandasamy \& Smarandache, 2009) and subgroup graphs (Anderson et al., 2012). Anderson et al. (2012) defining the subgroup graph of a group $G$ as a directed graph containing all elements of $G$ and two distinct vertices $x$ and $y$ will be joined by an arch if and only if $x y$ is belong to the related subgroup. When the given subgroup is a normal subgroup of $G$, then the 
subgroup graph obtained is an undirected graph and thus its complement is also an undirected graph (Kakeri \& Erfanian, 2015).

Abdussakir has determined detour energy of the complement of subgroup graphs of dihedral group. (2017). In this research, the formulae of color Laplacian and color signless Laplacian spectrum and energy of these graphs are determined.

\section{Literature Review}

Let $G$ be a finite graph with order $\|V(G)\|=p$ and size $\mid E(G) \|=q$. Two distinct vertices $x$ and $y$ are called adjacent if they are joined by an edge in $G$ or $x y \in E(G)$. The adjacency matrix $A(G)$ of $G$ is a matrix $A(G)=\left[a_{i j}\right]$ of order $p$ where $a_{i j}=1$ if $v_{i} v_{j} \in E(G)$ and $a_{i j}=0$ if $v_{i} v_{j} \notin E(G)$ (Abdussakir et al., 2009). The degree $\operatorname{deg}(x)$ of a vertex $x$ in $G$ is the number of vertices that adjacent with $x$. The degree matrix $D(G)$ of $G$ is matrix $D(G)=\left[d_{i j}\right]$ of order $p$ where $d_{i j}=\operatorname{deg}\left(v_{i}\right)$ for $i=j$ and $d_{i j}=0$ otherwise (Abdussakir et al., 2017).

Matrix $L(G)=D(G)-A(G)$ is called the Laplacian matrix of graph $G$ (Elvierayani \& Abdussakir, 2013) and matrix $L^{+}(G)=D(G)+A(G)$ is called the signless Laplacian matrix of $G$ (Ashraf et al., 2013). The characteristic polynomial of $L(G)$ and $L^{+}(G)$ are $\operatorname{det}(L(G)-\lambda I)$ and $\operatorname{det}\left(L^{+}(G)-\lambda^{+} I\right)$, respectively. The roots of the characteristic equation of a matrix are called eigenvalues of the matrix (Jog \& Kotambari, 2016). The eigenvalues of $L(G)$ are called Laplacian eigenvalues of $G$ and the eigenvalues of $L^{+}(G)$ are called signless Laplacian eigenvalues of $G$.

Let $\lambda_{1}, \lambda_{2}, \ldots, \lambda_{\mathrm{p}}$ are Laplacian eigenvalues of graph $G$. The Laplacian energy of $G$ is defined by Zhou \& Gutman (2007) as

$$
L E(G)=\sum_{i=1}^{\psi}\left|\lambda_{i}-\frac{m}{m}\right| .
$$

In similar way, the signless Laplacian energy of graph $G$ is defined by Xi \& Wang (2017) as

$$
L E^{+}(G)=\Sigma_{i=1}^{p}\left|\lambda T-\frac{m a g}{-n}\right|
$$

where $\lambda_{t}^{\dagger}$ are signless Laplacian eigenvalues of $G$.

Adiga et al (2013) introduced the concept of color energy of a graph motivated by the work of Sampathkumar and Sriraj (2013). Coloring of a graph $G$ is assigning color to all vertices of $G$ such that two adjacent vertices have the different color (Bondy \& Murty 2008). Coloring of graph $G$ can be considered as a function $c: V(G) \rightarrow \mathbb{N}$ such that $c(x) \neq c(y)$ if $x$ and $y$ are adjacent in $G$ (Chartrand et al. 2016). The minimum positive integer $k$ is called chromatic number of $G$ and denoted by $\chi(G)$ if $c \nabla V(G) \rightarrow\{1,2, \ldots, k\}$ is a coloring of $G$ (Akbari et al., 2009).

Let $G$ be a colored graph and $c$ is a coloring of $G$. Color matrix $A_{c}(G)$ of $G$ is defined by

$$
a_{i j}=\left\{-3, \text { if } v_{j} \text { and } w_{j} \text { are not adjacent and } o(v)=o(v)\right.
$$

Eigenvalues of $A_{C}(G)$ are called color eigenvalues of $G$ and the sum of absolute values of color eigenvalues of $G$ is called color energy of $G$ and denoted by $\boldsymbol{E}_{c}(\boldsymbol{G})$ (Adiga et al. 2013). Bhat and D'souza (2015) and also Shigehalli and Betageri (2015) introduced color Laplacian matrix of a colored graph $G$ as $L_{\sigma}(G)=D(G)-A_{\sigma}(G)$. Furthermore, Bhat and D'souza 
(2017b) defined color signless Laplacian matrix of a colored graph $G$ as $\mathcal{L}_{\varepsilon}(G)=D(G)+A_{\varepsilon}(G)$, where $D(G)$ is the degree matrix of $G$. The eigenvalues of $\mathbb{Z}_{\varepsilon}(G)$ are called color Laplacian eigenvalues of $G$ and the eigenvalues of $L_{\varepsilon}^{+}(G)$ are called color signless Laplacian eigenvalues of $G$.

In a similar way with the definition of Laplacian and signless Laplacian energy, the color Laplacian energy of colored graph $G$ of order $p$ and size $q$ is defined as

$$
L E_{c}(G)=\sum_{i=1}^{F}\left|u_{i}-\frac{-u_{i}}{n}\right|
$$

and its color signless Laplacian energy is defined as

$$
L E_{E}^{+}(G)=\sum_{i=1}^{F}\left|\mu_{i}^{+}-\frac{-u_{i}}{-}\right|
$$

where $\mu_{i}$ are color Laplacian eigenvalues and $\mu_{i}^{+}$are color signless Laplacian eigenvalues (Bhat \& D'souza, 2015, Shigehalli \& Betageri, 2015 and Bhat \& D'Souza, 2017b).

If a graph $G$ is colored with $X(G)$, then color matrix, color Laplacian matrix and color signless Laplacian matrix of $G$ are called chromatic matrix, chromatic Laplacian matrix and chromatic signless Laplacian matrix of $G$ and denoted by $A_{y}(G), L_{z}(G)$ and $L_{y}^{+}(G)$, respectively. Furthermore, chromatic Laplacian energy and chromatic signless Laplacian energy of $G$ are denoted by $L E_{y}(G)$ and $L E_{y}^{+}(G)$.

Let $\mu_{1}>\mu_{2}>\ldots>\mu_{3}(s \leq p)$ are distinct color Laplacian eigenvalues of $G$ and $m_{1}, m_{2}, \ldots, m_{g}$ are their multiplicities. The color Laplacian spectrum of $G$ is defined as

$$
\operatorname{LspeG}(G)=\left|\begin{array}{llll}
r+ & r- & \cdots & r r \\
m . & m- & & m
\end{array}\right| .
$$

Let $\mu_{1}^{+}>\mu_{2}^{+}>\ldots>\mu_{t}^{+}(t \leq p)$ are distinct color Laplacian eigenvalues of $G$ and $m_{1}^{+}, m_{2}^{+}, \ldots, m_{t}^{+}$are their multiplicities. The color signless Laplacian spectrum of $G$ is defined as

$$
\operatorname{Lspec}_{c}+(G)=\left|\begin{array}{llll}
m_{1} & n z & \cdots & n_{\tau} \\
\ldots+ & \ldots+ & \ldots+
\end{array}\right|
$$

The following are previous results that will be used in further discussion.

Theorem 2.1. (Bhat \& D'souza 2015) For $n \geq 2$, then

$$
\begin{gathered}
\operatorname{Lspec}_{X}\left(K_{Y}\right)=\mid{ }_{n} \text {, }, \frac{1}{1} \mid \\
\text { and } \\
L E_{X}\left(K_{n}\right)=2(n-1) .
\end{gathered}
$$

Theorem 2.2. (Bhat \& D'souza 2015) For $n \geq 1$, then

$$
\begin{gathered}
\operatorname{Lspec}_{y}\left(\mathbb{N}_{n}\right)=\left|\begin{array}{ll}
n+1 & -+1 \\
1 & \ldots
\end{array}\right| \\
\text { and }
\end{gathered}
$$




$$
L E_{y}\left(\mathbb{N}_{n}\right)=2(n-1)
$$

Theorem 2.3. (Bhat \& D'souza 2015) The chromatic Laplacian spectrum of $K_{\mathrm{m}, \mathrm{n}}$ is

$$
\begin{aligned}
& |m+n-1+\sqrt{ } m m+n-1-\sqrt{ } m n-1 \quad n-1| \\
& \text { and } \\
& L E_{y}\left(K_{m, n}\right)=\frac{2}{m+n}\left[m^{2}+n^{2}+(m+n)(\sqrt{m n}-1)\right], \\
& \text { if } m=n \text { and } n=m+1 \text { and } \\
& L E_{X}\left(K_{m, n}\right)=\frac{-\pi m a}{m+n}[(n-m) / m n+(m+n)] \text {, } \\
& \text { if } n>m+1 \text {. }
\end{aligned}
$$

Theorem 2.4. (Bhat \& D'Souza 2017b) For $n \geq 2$,

$$
\begin{gathered}
\operatorname{Lspec}_{X}^{+}\left(K_{n}\right)=\left|\begin{array}{ccc}
-n-1, & n-4
\end{array}\right| \\
\text { and } \\
L E_{Y}^{+}\left(K_{n}\right)=2(n-1) .
\end{gathered}
$$

Theorem 2.5. (Bhat \& D'Souza 2017b) For $n \geq 1$, then

$$
\begin{gathered}
\operatorname{Lspec}+\left(\mathrm{N}_{n}\right)=\mid{ }^{+}-{ }_{n}-+1 \\
\text { and } \\
L E_{X}^{+}\left(\mathrm{N}_{n}\right)=2(n-1) .
\end{gathered}
$$

Theorem 2.6. (Bhat \& D'Souza 2017b) The chromatic signless Laplacian spectrum of $K_{m_{p} \mathrm{n}}$ is

$$
\begin{aligned}
& \left|\begin{array}{llll}
m+1 & n+1 & 1+\sqrt{ } m^{2}+n^{2}-m n & 1-\sqrt{ } m^{2}+n^{2}-m n
\end{array}\right| \\
& \text { and } \\
& L E_{Y}^{+}\left(K_{m n}\right)=2\left(\sqrt{ } m^{2}+n^{2}-m n-1\right)+\frac{\ldots n}{n+1}
\end{aligned}
$$

Recently, graph of group has also been a research topic discussed by many researchers. Anderson et al (2012) introduced the concept of subgroup graph of a group. Let $H$ is any subgroup of a group $G$. The subgroup graph of $G$ is defined as a directed graph with vertex set $G$ such that vertex $x$ will be adjacent to vertex $y$ if and only if $x \neq y$ and $x y \in H$ and denoted by $\Gamma_{H}(G)$. If $H$ is a normal subgroup of $G$, then $\Gamma_{M}(G)$ is an undirected simple graph and so $\overline{\Gamma_{H}(G)}$ is an undirected simple graph too (Kakeri \& Erfanian, 2015).

Research on color Laplacian and color signless Laplacian spectrum and energy of subgroup graphs have not reported yet until now, especially for the subgroup graphs of 
dihedral group. Let $D_{M M}=\langle r, s\rangle$ is the dihedral group of order $2 n(n \geq 3)$. All normal subgroup of $D_{z n}$ are the subgroups $\langle r\rangle,\left\langle r^{d}\right\rangle$ where $d$ is divisor of $n$, and $D_{n n}$, for odd $n$ and the subgroups $\langle r\rangle,\left\langle r^{d}\right\rangle$ where $d$ is divisor of $n,\left\langle r^{2}, s\right\rangle,\left\langle r^{2}, s\right\rangle$ and $D_{n n}$, for even $n$ (Abdussakir, 2017). Motivated by this condition, the color Laplacian and color signless Laplacian spectrum and energy of subgroup graphs of dihedral group are studied. All subgroups discussed in this paper are normal subgroups of $D_{2 n}$.

\section{Results}

Because this study focused on the coloring with minimum number of colors, the main results of this study are the chromatic Laplacian spectrum and energy and the chromatic signless Laplacian spectrum and energy.

$$
\begin{aligned}
& \text { Theorem 3.1. For } n \geq 3 \text {, then } \\
& \operatorname{Lspec}_{X}\left(\Gamma_{D_{3 n}}\left(D_{2 n}\right)\right)=\mid \begin{array}{l}
2_{n n}+1 \\
\text { and }
\end{array} \\
& L E_{X}\left(\Gamma_{D_{2 n}}\left(D_{2 n}\right)\right)=2(2 n-1) .
\end{aligned}
$$

Proof. By definition of subgroup graph, then the subgroup graph $\Gamma_{D_{n z}}\left(D_{2 n}\right)$ of dihedral group

$D_{z n}$ is a complete graph of order $2 n$. By Theorem 2.1, the proof is obtained.

$$
\begin{aligned}
& \text { Theorem 3.2. For } n \geq 3 \text {, then } \\
& \operatorname{Lspec}_{Y}\left(\mathrm{I}_{D_{n+1}}\left(D_{n n}\right)\right)=\left.\right|^{2 n} \text { and } \\
& L E_{Y}\left(\Gamma_{D_{3 n}}\left(D_{2 n}\right)\right)=2(2 n-1) .
\end{aligned}
$$

Proof. Since $\Gamma_{D_{n n}}\left(D_{2 n}\right)$ is a complete graph of order $2 n$, then $\Gamma_{D_{n n}}\left(D_{2 n}\right)$ is a null graph of order $2 n$. Using Theorem 2.2 the proof is obtained.

$$
\begin{aligned}
& \text { Theorem 3.3. For } n \geq 3 \text {, then }
\end{aligned}
$$

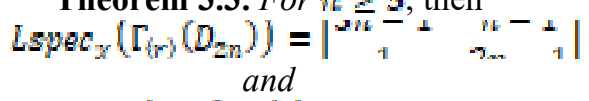

$$
\begin{aligned}
& L E_{y}\left(\Gamma_{i n}\left(D_{2 n}\right)\right)=2(2 n-1) \text {. }
\end{aligned}
$$

Proof. The subgroup graph $\Gamma_{i n}\left(D_{2 n}\right)$ of dihedral group $D_{2 n}$ is an unconnected graph with two components. The two components are complete graphs of order $n$ with vertex set

$\left\{1, r, r^{2}, \ldots, r^{n-1}\right\}$ and $\left[s, s r_{1} s r^{2}, \ldots, s r^{n-1}\right]$, respectively. Therefore, $\mathbb{T}_{(w)}\left(D_{M N}\right)$ is a complete bipartite graph $K_{n, n}$. By Theorem 2.3 and some computation, the proof is obtained.

$$
\begin{aligned}
& \text { Theorem 3.4. For } n \geq 4 \text { and } n \text { is even, then }
\end{aligned}
$$

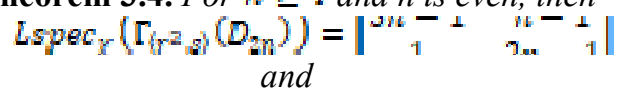

$$
\begin{aligned}
& L E_{y}\left(\Gamma_{i r_{3} z_{3}}\left(D_{2 n}\right)\right)=2(2 n-1) .
\end{aligned}
$$

Proof. The normal subgroup $\left\langle n^{2}, 3\right\rangle$ of $D_{2 n}$ for $n \geq 4$ and $n$ is even is

$$
\left\langle r^{2}, s\right\rangle=\left\{1, r^{2}, r^{4}, \ldots, r^{n-2}, s, s r^{2}, s r^{4}, \ldots, s v^{n-2}\right\}
$$

and $\left(s^{k} r^{2}\right)\left(s^{k} r^{2}\right) \in\left\langle r^{2}, s\right\rangle$ if and only if $i$ and $j$ both even or both odd, for $1 \leq i, j \leq n-2$ and $k=0,1$. Then, the subgroup graph $\mathbb{T}_{i r^{z}, g}\left(D_{2 M}\right)$ has two components and each component is 
complete graph $K_{n}$ of order $n$. Therefore, $\mathbb{T}_{\left(r^{z}, g\right.}\left(\mathbb{O}_{2 n}\right)$ is a complete bipartite graph $K_{n n}$. By using Theorem 2.3, the proof is complete.

$$
\begin{aligned}
& \text { Theorem 3.5. For } n \geq 4 \text { and } n \text { is even, then }
\end{aligned}
$$

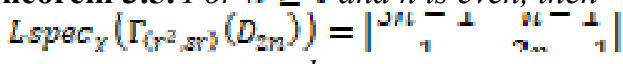

$$
\begin{aligned}
& \text { and } \\
& L E_{y}\left(T_{\left(r^{2}, g r\right.}\left(D_{2 n}\right)\right)=2(2 n-1) .
\end{aligned}
$$

Proof. The subgroup graph $\Gamma_{\left(\mathrm{r}^{2} \mathrm{gr}\right.}\left(D_{n \mathrm{n}}\right)$ has two components and each component is complete graph $K_{n}$ of order $n$. Therefore, $\Gamma_{\left\{r^{2}, s r\right.}\left(D_{n n}\right)$ is a complete bipartite graph $K_{n n}$. The proof is obvious by using Theorem 2.3.

$$
\begin{aligned}
& \text { Theorem 3.6. For } n \geq 3 \text {, then }
\end{aligned}
$$

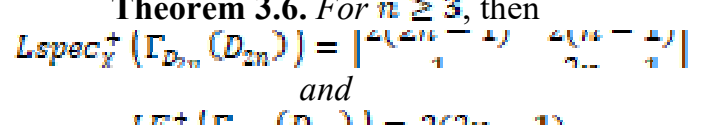

$$
\begin{aligned}
& L E_{Y}^{+}\left(\Gamma_{D_{n}}\left(D_{n}\right)\right)=2(2 n-1) \text {. }
\end{aligned}
$$

Proof. Since the subgroup graph $\Gamma_{D_{n}}\left(D_{n n}\right)$ of dihedral group $D_{n n}$ is a complete graph of order $2 n$, by using Theorem 2.4 the proof is obtained.

$$
\begin{gathered}
\text { Theorem 3.7. For } n \geq 3 \text {, then } \\
\operatorname{Lspec}_{Y}^{+}\left(\Gamma_{D_{n}}\left(D_{n=1}\right)\right)=\left.\right|_{3}-1 y \\
\text { and } \\
L E_{y}^{+}\left(\Gamma_{D_{n}}\left(D_{2 n}\right)\right)=2(2 n-1) .
\end{gathered}
$$

Proof. Since $\Gamma_{D_{n n}}\left(D_{2 n}\right)$ is a complete graph of order $2 n$, we have $\Gamma_{D_{3 n}}\left(D_{2 n}\right)$ is a null graph of order $2 n$. Using Theorem 2.5 the proof is obtained.

$$
\begin{aligned}
& \text { Theorem 3.8. For } n \geq 3 \text {, then }
\end{aligned}
$$

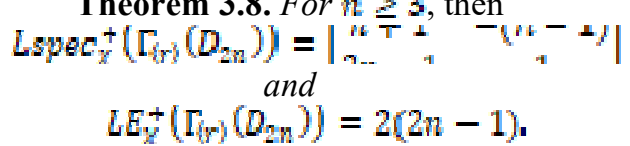

Proof. Since $\Gamma_{(n)}\left(D_{n n}\right)$ is a complete bipartite graph $K_{n n}$. By Theorem 2.6 and some computations, the desired proof is obtained.

Theorem 3.9. For $n \geq 4$ and $n$ is even, then

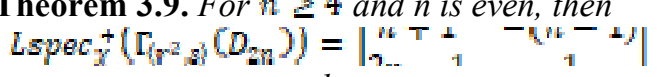

$$
\begin{aligned}
& L E_{Y}^{+}\left(T_{\left(r_{n}\right)^{3}}\left(D_{n}\right)\right)=2(2 n-1) \text {. }
\end{aligned}
$$

Proof. By the proof of Theorem 3.4. then $\Gamma_{\left\langle r^{2}, z\right\}}\left(D_{2 n}\right)$ is a complete bipartite graph $K_{n n}$. By using Theorem 2.5 the proof is obtained.

Theorem 3.10. For $n \geq 4$ and $n$ is even, then

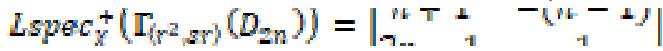

$$
\begin{aligned}
& \text { and } \\
& L E_{X}^{+}\left(\Gamma_{r^{2}, \operatorname{sr} 2}\left(D_{2 n}\right)\right)=2(2 n-1) \text {. }
\end{aligned}
$$

Proof. Since $\Gamma_{\left(\mathbb{Z}_{N W}\right)}\left(D_{n}\right)$ is a complete bipartite graph $K_{n n}$ by the proof of Theorem 3.5, then it is obvious by using Theorem 2.6 .

\section{Conclusions}


The formulae of chromatic Laplacian and chromatic signless Laplacian spectrum and energy of complement of subgroup graphs of dihedral group for several normal subgroups have been determined. Further research is needed to observe chromatic Laplacian and chromatic signless Laplacian spectrum and energy of complement of subgroup graphs of dihedral group for the rest normal subgroups.

Acknowledgements. We would like to thank Faculty of Science and Technology, State Islamic University of Malang for partial support to this research.

\section{References}

[1] Abdussakir, 2017. Detour energy of complement of subgroup graph of dihedral group. ZeroJurnal Sains, Matematika dan Terapan, 1(2), pp.41-48.

[2] Abdussakir, Azizah, N.N. \& Nofandika, F.F., 2009. Teori graf: Topik dasar untuk tugas akhir/skripsi, Malang: UIN Malang Press.

[3] Abdussakir, Elvierayani, R.R. \& Nafisah, M., 2017. On the spectra of commuting and non commuting graph on dihedral group. Cauchy-Jurnal Matematika Murni dan Aplikasi, 4(May), pp.176-182.

[4] Adiga, C. et al., 2013. Color energy of a graph. In Proc. Jangjeon Math. Soc. pp. 335-351.

[5] Adiga, C. \& Smitha, M., 2009. On maximum degree energy of a graph. Int. J. Contemp. Math. Sci, 4(5-8), pp.385-396.

[6] Akbari, S., Ghorbani, E. \& Zare, S., 2009. Some relations between rank, chromatic number and energy of graphs. Discrete Mathematics. 309(3), pp.601-605.

[7] Alfuraidan, M.R. \& Zakariya, Y.F., 2017. Inverse graphs associated with finite groups. Electronic Journal of Graph Theory and Application, 5(1), pp.142-154.

[8] Anderson, D.F., Fasteen, J. \& Lagrange, J.D., 2012. The subgroup graph of a group. Arab Journal of Mathematics, 1, pp.17-27.

[9] Ashraf, F., Omidi, G.R. \& Tayfeh-Rezaie, B., 2013. On the sum of signless Laplacian eigenvalues of a graph. Linear Algebra and Its Applications, 438, pp.4539-4546.

[10] Ayyaswamy, S.K. \& Balachandran, S., 2010. On detour spectra of some graphs. International Journal of Mathematical, Computational, Physical, Electrical and Computer Engineering, 4(7), pp.1038-1040.

[11] Balakrishnan, R., 2004. The energy of a graph. Linear Algebra and Its Applications, 387, pp.287-295.

[12] Bhat, P.G. \& D'souza, S., 2015. Color Laplacian energy of a graph. Proceedings of the Jangjeon Mathematical Society, 18(3), pp.321-330.

[13] Bhat, P.G. \& D'Souza, S., 2017a. Color signless Laplacian energy of graphs. AKCE International Journal of Graphs and Combinatorics, 14(2), pp.142-148.

[14] Bhat, P.G. \& D'Souza, S., 2017b. Color signless Laplacian energy of graphs. AKCE International Journal of Graphs and Combinatorics, 14(2), pp.142-148.

[15] Bondy, J.A. \& Murty, U.S.R., 2008. Graph theory. New York: Springer.

[16] Chartrand, G., Lesniak, L. \& Zhang, P., 2016. Graphs and digraphs 6th ed., Florida: CRC Press.

[17] Chelvam, T.T., Selvakumar, K. \& Raja, S., 2011. Commuting graphs on dihedral group main results. The Journal of Mathematics and Computer Science, 2(2), pp.402-406.

[18] Das, K.C., Sorgun, S. \& Xu, K., 2014. On Randic energy of graphs. MATCH Commun. Math. Comput. Chem, 72, pp.227-238.

[19] Elvierayani, R.R. \& Abdussakir, 2013. Spectrum of the Laplacian matrix of non-commuting graph of dihedral group D2n. In Proceeding of International Conference The 4th Green Technology. Malang: Faculty of Science and Technology, pp. 321-323. 
[20] Erfanian, A. \& Tolue, B., 2012. Conjugate graphs of finite groups. Discrete Mathematics, Algorithms and Applications, 04(02), pp.1-8.

[21] Güngör, D.A. \& Çevik, A.S., 2010. On the Harary energy and Harary Estrada index of a graph. MATCH Commun. Math. Comput. Chem., 64(1), pp.281-296.

[22] Gutman, I. et al., 2009. On incidence energy of a graph. Linear Algebra and Its Applications, 431(8), pp.1223-1233.

[23] Gutman, I., 2001. The energy of a graph: old and new results. In Algebraic Combinatorics and Applications. Berlin, Heidelberg: Springer, pp. 196-211.

[24] Gutman, I., 1978. The energy of a graph. Ber. Math-Statist. Sekt. Forschungszentrum Graz, $103, \mathrm{pp} .1-22$.

[25] Gutman, I. \& Wagner, S., 2012. The matching energy of a graph. Discrete Applied Mathematics, 160(15), pp.2177-2187.

[26] Gutman, I. \& Zhou, B., 2006. Laplacian energy of a graph. Linear Algebra and Its Applications, 414, pp.29-37.

[27] Heydemann, M.-C., 1997. Cayley graphs and interconnection networks. In Graph Symmetry. Dordrecht: Springer, pp. 167-224.

[28] Jog, S.R. \& Kotambari, R., 2016. On the adjacency, Laplacian, and signless Laplacian spectrum of coalescence of complete graphs. Journal of Mathematics, 2016, pp.1-11.

[29] Jooyandeh, M., Kiani, D. \& Mirzakhah, M., 2009. Incidence energy of a graph. MATCH: Communication in Mathematical and in Computer Chemistry, 62, pp.561-572.

[30] Kakeri, F. \& Erfanian, A., 2015. The complement of subgroup graph of a group. Journal of Prime Research in Mathematics, 11, pp.55-60.

[31] Kandasamy, W.B.V. \& Smarandache, F., 2009. Groups as graphs, Judetul Olt, Romania: Editura CuArt.

[32] Kanna, M.R.R., Dharmendra, B.N. \& Kumar, R.P., 2013. Minimum covering distance energy of a graph. Applied Mathematical Sciences, 7(111), pp.5525-5536.

[33] Kanna, M.R.R., Dharmendra, B.N. \& Sridhara, G., 2014. Minimum dominating distance energy of a graph. Journal of Indonesian Mathematics Society, 20(1), pp.19-29.

[34] Kelarev, A. V \& Praeger, C.E., 2003. On transitive Cayley graphs of groups and semigroups. European Journal of Combinatorics, 24(1), pp.59-72.

[35] Liu, Z., 2010. Energy, Laplacian energy and Zagreb index of line graph, middle graph and total graph. Int. J. Contemp. Math. Sci., 5(18), pp.895-900.

[36] Ramane, H.S. et al., 2008. Estimating the distance energy of graphs. Graph Theory Notes of New York, 55, pp.27-32.

[37] Raza, Z. \& Faizi, S., 2013. Non-commuting graph of a finitely presented group. Sci. Int. (Lahore), 25(4), pp.883-885.

[38] Sampathkumar, E. \& Sriraj, M.A., 2013. Vertex labeled/colored graphs, matrices and signed graphs. Journal of Combinatorics, Information \& System Sciences, 38(1-4), pp.113-120.

[39] Shigehalli, V.S. \& Betageri, K.S., 2015. Color Laplacian Energy of Graphs. Journal of Computer and Mathematical Sciences, 6(9), pp.485-494.

[40] Xi, W. \& Wang, L., 2017. Bounds for the signless Laplacian energy of digraphs. Indian Journal of Pure and Applied Mathematics, 48(3), pp.411-421.

[41] Zhou, B. \& Gutman, I., 2007. On Laplacian energy of graphs. MATCH Commun. Math. Comput. Chem, 57, pp.211-220.

[42] Zhou, B., Gutman, I. \& Aleksi, T., 2008. A note on Laplacian energy of graphs. MATCH Commun. Math. Comput. Chem, 60, pp.441-446. 